\title{
Limiting the Excess Broadcast in Multipath Routing Using Ant Colony Optimization
}

\author{
Amanpreet Kaur ${ }^{1}$, Gurpreet Singh ${ }^{2 *}$, Rohan Gupta ${ }^{3}$ \\ ${ }^{I}$ Assistant Professor, Chandigarh University, Gharuan, Mohali, Punjab, India. \\ ${ }^{2}$ Associate Professor, Punjab Institute of Technology, Rajpura, Punjab, India. \\ ${ }^{3}$ Research Scholar, ECE Dept, IKG Punjab Technical University, Kapurthala, Punjab, India. \\ *Corresponding author E-mail: gps_ynr@yahoo.com
}

\begin{abstract}
Single path routing in mobile adhoc network (MANET) suffers from frequent disconnections due to movement of nodes. So, multipath routing evolves as a solution to the single path routing. Many researchers are working on devising multipath routing protocols with new features and strategies. Ant colony optimization is also used by many investigators for developing new solutions for multipath routing in MANET. In this paper we have worked on controlling the broadcast being done by the intermediate nodes. We improved on our work done in Amanpreet Kaur et al. titled "ACO Agent Based Routing in AOMDV Environment" by making use of channel contention and backoff technique. We compared the newly designed algorithm with three broad categories unipath, multipath and ant based algorithm. The new algorithm performs better as compared with other algorithms on the basis of various QoS metrics like throughput, jitter, end to end delay, and number of packets send, lost in packets and delivery ratio.
\end{abstract}

\section{Introduction}

The Mobile ad hoc networks (MANETs) are special category of connectionless network that forms provisional data connections for the viewpoint of communication. MANET is a infrastructureless and regularly self-configuring network in which mobility based devices connect with each other without wires [1]. In MANET, each device can travel in any direction independently. MANET does not have a central coordination point or a certain topology. To send and receive packets in MANETs are not as easy as in infrastructure based networks. Now days there is rapid increase in applications used in wireless network due to PDAs, mobile phones, laptops etc. Supporting the mobility is one of the issue in wireless networks [2]. In such networks nodes exploit the exact random access non wire channel. The host nodes inside wireless network behave like routers that are capable of routing data to rest of the nodes in the network. There is no support for infrastructure in MANET, there is no infrastructure support. In MANET, a destination node or any intermediate node might not be in range of a source node who is generating packets, therefore a proper procedure for routing is indeed to find the path, so that data can be forwarded appropriately in between source and the destination. Routing in MANET can be categorized into three distinctive classes under the name of Reactive, Proactive and Hybrid techniques. In the proactive natured routing, the routing tables are maintained by every node in a network. These routing tables are reorganized on regular basis [1]. A broadcast message is send by every node in the network to identify any alteration in the topology of the network. When a sender is in necessity to forward data packet to target node and if a route is already exist in the routing table before traffic to start then transmission occurs without any delay. These protocols obtain additional overhead cost due to maintaining up-to-date information [6]. In Reactive Routing Protocol, each node works on-demand i.e. path is searched when there is need to communicate the data between peers. Each Node floods a control message by global broadcast when it starts discovering a route [2]. The key benefit of ondemand protocols is that it needs fewer routing information as compare to others but the drawbacks are that it produces large control overhead due to discovery of the route during the change in topology which occurs frequently in MANETs. This routing protocol obtains higher latency [6]. Hybrid Routing Protocol is a mixture of both proactive as well as reactive protocols taking the best features from both of above protocols.

Ant colony optimization (ACO) indicates the practice used now days for the optimization problems. ACO is commonly used for solving problems involving computational complexities which can be reduced to finding best path [3] [18]. The inspirational basis of any ACO is the nature of the working of real ant colonies. This foraging behavior of real ants is copied by the artificial ant colonies in finding the approximate solutions. Ants deposit pheromone while traveling from the nest to food and food to nest back. This pheromone value used to communicate with one another to find shortest path [5] [19].

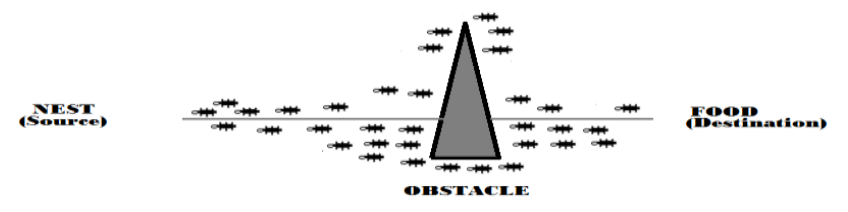

Fig. 1: Ant find shortest path while searching food

Pheromone aggregation works faster on the shorter path as compare to longest path. Ant deposits a certain amount of pheromone 
while travelling and each ant probabilistically follows a direction in which ant found pheromone value is high. This behavior of ants can be used to explain how they can find the least cost path from their shell to source of food as shown in figure 1.

\section{Traditional Protocols}

\subsection{AODV}

Ad hoc On-Demand Distance Vector routing protocol is betrothed for utilization by moving items in the temporary network. AODV tenders fast revision to vibrant conditions of link, low network utilization, overhead due to memory, low processing and determines single path in the ad hoc network. AODV uses sequence numbers issued with reference to destinations to ensure freedom from loop each time by avoiding problems such as "counting to infinity" connected with old method of distance vector algorithms [6] [17]. AODV algorithm facilitates self-starting and dynamic multi-hop routing among the contributed nodes willing to set up and sustain a temporary network. AODV permits moving entities to rapidly create ways for fresh targets. In AODV nodes do not need to maintain routes for those destinations which are not participating in ongoing conversation. It facilitates moving nodes to react to breakages in links and the changing in topology. AODV make use the sequence number inspired by destination to recognize the latest path. Source node as well as intermediate node keeps the information for the next hop associated to every data flow of different packet transmission [14]. When there is breakage in links, AODV notifies the affected set of nodes so that they can vitiate the paths used by the lost link. Control messages are used in AODV for Route Discovery. Route Request packet (RREQ) and Route Reply packet (RREP) are used when a source node desires to forward packet towards the target node. RREQs to send to neighbors in the network using broadcast method. The forward path is created while travelling through intermediate nodes which is helpful in maintaining routing table. The destination node send route error (RERR) packets towards the source node on path break. On receiving RERR, the source node initiate again the discovery of path.

\subsection{Ant-AODV}

Ant-AODV is effective technique, which creates a combination of both AODV routing protocols and ant-based routing. This combination tries to surmount the inborn drawbacks of other traditional protocols. This hybrid methodology enhances the connectivity between nodes and also decreases the latency in route discovery and reduces end-to-end delay. In routing techniques which are dependent on ants' activity, route establishment is reliant on the moving ants [9]. These ants visit the node one by one and provide best route towards destination. Whenever a node does not have a route and any node who desires to transmit the data packets to the target node, the sender will have to maintain the information of data packets in the memory of ant till that particular ant finishes its journey at the destination node. Whenever the ant reaches to its target node then ant utilizes the information about history for modifying the routing table [11]. More overhead is likely to occur if the history size increases. All nodes data present inside the network depend on the ants for imparting the node the information about routing. In Ant-AODV, the node connectivity is improved by the use of ants which also reduces the time consumed in the discovery of route. On the launch the RREQ by source, the likelihood of getting the responses swiftly from the nearby nodes increases. In Ant-AODV, the ants make update to the paths constantly and sender can shift transferring data from a lengthy route to a newer route which is smaller route. Ant-AODV employs the route error messages to indicate the nodes in upstream in link failure. Ant-AODV utilizes routing table which is used by both AODV packets and ants packet [4]. Continually HELLO messages are broadcast to other nodes to uphold the neighbor table. This neighbor table can be used to choose the arbitrarily chosen next hop from the neighbors list by the artificial ant.

\subsection{DSR}

Dynamic Source Routing protocol is very straightforward and competent algorithm for routing. DSR has been created particularly for applying in mobile ad hoc networks with multi-hop nature in traversing. DSR completely is of self-managing and selfarranging nature. DSR works without the need for any pre-existing network administration or infrastructure [7]. Mainly two components are used in the MANET; Route Discovery and Route Maintenance. The process of Route Discovery checks or determines the optimal path for a transmission of packet between the couple of source node and destination node. Route Maintenance process make certain that the path being used for transmission relics optimal and free from loops [13]. Route Maintenance technique is a process in which the source node is proficient to maintain the optimal or best path even if there is a change in network topology and preceding link has been altered; in this situation it cannot utilize its route to target node because the linkage of that path will not work now in the network. When route maintenance phase specify the sender node about breakage in link then sender node tries to utilize alternative path, or it may initiate the route discovery process over again to identify a fresh path for successive transmission of packets.

\subsection{AOMDV}

Ad hoc on-demand Multipath Distance Vector routing protocol broadens AODV to a multipath routing protocol [8]. Whenever, a routing protocol endeavors to find multiple routes towards destination, it is useful many times to have a measure of the similarity of routes [10]. There are classifications of routes according to the routes similarity:-

1. Node Disjoint routes are routes in which no nodes or links are common between paths excluding the source node and the destination node.

2. Link Disjoint routes don't have any link common between the selected paths, but the routes might have some common nodes.

3. Non-disjoint paths may have either common links or common nodes.

An Ad hoc On-demand Multipath Distance Vector routing protocol produces link disjoint paths by using a property of flooding. Authors describe the property and its proof. Whenever, a node in the AOMDV network receives multiple copies of a Route Request (RREQ) packet with the same sequence number, then node processes all received copies, since these copies may contain different routes to the source. However, this may create transmission loops [15]. If node 1 floods a RREQ packet in the network, it is received and rebroadcast by another node 2 , and node 1 receives the packet again, then node 1 should not create a route from this packet. AOMDV uses the packet's sequence number and hop count to impose loop freedom.

\subsection{MDART}

The Multi-Path Dynamic Addressing Routing (MDART) is a proactive routing protocol and keeps information about available routes. MDART is inspired from the well-known shortest path DHT-based routing protocol known as DART. MDART is a multipath protocol. MDART is capable in improving the charity of the address space against mobility based on tree [10]. MDART has two main features as compare to other multi-path routing protocol:-

- MDART are guaranteed to be coordination- free and communication- free route discovery. MDART announcement though does not need any additional coordination and overhead due to communication. 
- $\quad$ MDART uses dynamic addressing and it searches complete redundant routes available from the source to the destination. In MDART, the full routing procedure is dependent on the addresses of the transient network. The process should be sufficiently spread across the complete network. The network addresses and mapping between the nodes identity is given by a creation of Dynamic Hash Table.

\subsection{A-AOMDV-CB}

Ant Based-Adhoc on Demand Multipath Routing Protocol with controlled broadcast (A-AOMDV-CB) is a innovative algorithm inspired by the process of swarm intelligence. It uses the method adopted by the algorithms of ant colony optimization (ACO) with the features of multipath routing in the adhoc environment. The protocol uses two types of ants. One in short is called the FANT for forward ant and the other in short is called BANT for backward ant. FANT is produced at the node of sender and moves to the path of the receiver node to identify the way. When FANT has finished its journey and arrives at the destination, it may go dead and then BANT is formed at this point at the destination node and travel back to the source nod. BANT chases the path in reverse that was generated by the FANT. The FANT opts for the next node by calculating the probability $\left(\Omega_{\mathrm{ijd}}\right)$ which is evaluated as in equation (1). An ant moving towards node $d$ currently present at node $i$ selects the subsequent node $j$ by calculating the probability $\left(\Omega_{\mathrm{ijd}}\right)$ as follows:

$\Omega_{\mathrm{ijd}}=\frac{\rho_{i j d}+(\operatorname{Cos} \alpha) \theta \Gamma_{i j}}{(\operatorname{Cos} \alpha) \theta\left|N_{i}\right|+(1-\theta(\operatorname{Cos} \alpha))}$

Where $d$ is the target node, ${ }^{i}$ is the node where ant is presently residing, $j_{\text {is the next node, }} \theta$ is used as a heuristic function and $\rho_{i j d}$ is the value of the pheromone. $v_{1}$ and $v_{2}$ are the vectors with axial orientation to calculate the $\operatorname{cosine}$ value $\operatorname{Cos} \alpha$. $\left|N_{i}\right|$ shows the present numeral for all neighbours for node $i$ and $\Gamma_{\mathrm{ij}}$ represent heuristic value which ahead reliant on the queue length over the link $i-j$ and is evaluated as:

$\Gamma_{i j}=1-\left(\lambda_{i j} / \sum_{i=1}^{|N i|} \lambda_{i j}\right)$

$$
\operatorname{Cos} \alpha=\frac{v_{1} \cdot v_{2}}{\left|v_{1}\right| \cdot\left|v_{2}\right|}
$$

In recent ant based routing algorithms, the FANTs are routed in periodic fashion from source node and are further broadcasted by the subsequent nodes to their neighbour nodes. This algorithm controls this broadcast based on the maximum number of hops to destination by holding the packet for the certain time $T_{h}$ as follows:

$$
T_{h}=\frac{T D_{r e q}-T_{e}}{\left[M A X\left(m_{k}\right)+1\right]}
$$

$m_{k}$ is the highest number of nodes' hop for reaching to the sink for each intermediate node $k, T D_{r e q}$ is the time for delay requirement, $T_{e}$ is the time elapsed since the start of the packet,
$T_{W}$ is the wake-up interval and $C C B$ is the time period for channel contention and back off.

If a chosen node is previously exist in the path, then this path is rejected and is dropped. But, if chosen node has not selected before then this node is selected for forwarding the data or control packets. The quantity of the pheromone is increased on the favourable paths with the help of equation 5. However the quantity of pheromone is decreased on unused paths as per equation 6 :

$$
\begin{aligned}
& \rho(\mathrm{i})=\rho(\mathrm{i})+\mathrm{r}^{*}(1-\rho(\mathrm{i}))+\mathrm{r}^{2} * \rho_{\text {high }} / \mathrm{C} \\
& \rho(\mathrm{i})=\rho(\mathrm{i})-\mathrm{r} * \rho(\mathrm{i})-\mathrm{r}^{2} * \rho_{\text {high }} /\left(\mathrm{C}^{*}\left(\left|\mathrm{~N}_{\mathrm{i}}\right|-1\right)\right)
\end{aligned}
$$

Here $\rho \rho_{\text {high }}$ shows the highest value of obtained pheromone till far in the loop. $|\mathrm{Ni}|$ represents the number of neighbour nodes of node $i, C$ is a constant and depends on the dimension of time used in the experiment. In current paper the value of $C$ is taken as 1000 because simulation time is taken in milliseconds in experiment. $r$ is reinforcement factor that ranges from $(0,1]$.

Data structure has been made and accumulated as agent in AOMDV to corporate the ACO arrangement is made in AAOMDV-CB in NS-2 using $\mathrm{C} / \mathrm{C}++$ library with the following criteria of selection:

- A-AOMDV picks only two best paths having top value for the pheromone among the several identified paths.

- This algorithm uses link disjoint paths.

- Path selection may change on observing a positive change in the value of the pheromone over other paths.

- If any path breaks among the best two paths, then the new path is selected from the reserved path list or new selection will be made.

- Packet forwarding is controlled by introducing packet hold time by intermediate nodes.

Out of date routes can be identified and can be dealt with the help of revised value of the pheromone over paths.

\section{Simulation and Results}

For verifying the above algorithm, the simulation was carried in network simulator (NS-2) version 2.35. A network of 20 nodes was created for this purpose. A simulation script was written in TCL language for obtaining the network traces. The same script was executed for DSR, MDART, AOMDV, ANT-AODV and AAOMDV-CB. The results were extracted from the trace files and a comparison table is created as shown in table 1 for various performance metrics. The data in this table are used to create the graphs for various performance metrics.

Table 1: Data table for various Performance Metrics

\begin{tabular}{|c|c|c|c|c|c|}
\hline Performance Metrics & A-AOMDV-CB & Ant-AODV & AOMDV & MDART & DSR \\
\hline Throughput (Bvtes/ Sec) & 55950.5 & 54758 & 55503.5 & 51379 & 49168.5 \\
\hline Packets Send & 4601 & 4216 & 4582 & 3958 & 3787 \\
\hline Packets Drop & 0 & 3 & 1 & 1 & 10 \\
\hline Delivery Ratio & 100 & 99.928843 & 99.97818 & 99.97473 & 99.73594 \\
\hline Average litter & .124426881 & .2930803 & .138538 & .178871 & .460178 \\
\hline Average End to End Delay & .292174898 & .461926 & .29017 & .297864 & .647297 \\
\hline
\end{tabular}




\subsection{Throughput}

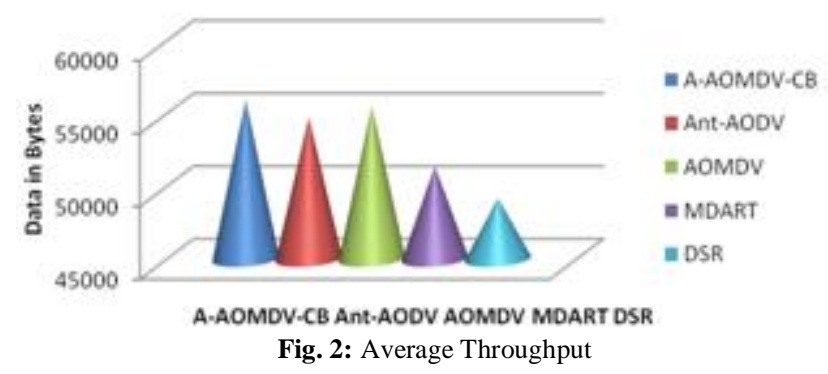

It is evaluated as the number of packets communicated per unit time. We can sum up the total data for the complete simulation time and divide it by the total time to get average throughput. The graph given below predicts the average throughput in bytes per second. When evaluated on the factor of average throughput, the graph depicts that A-AOMDV-CB performs higher than other four algorithms.

\subsection{Numbers of Packet Send}

This parameter evaluates the efficiency of an algorithm to send the more data in same spam of time. Larger value in this parameter indicates the better efficiency of algorithm.

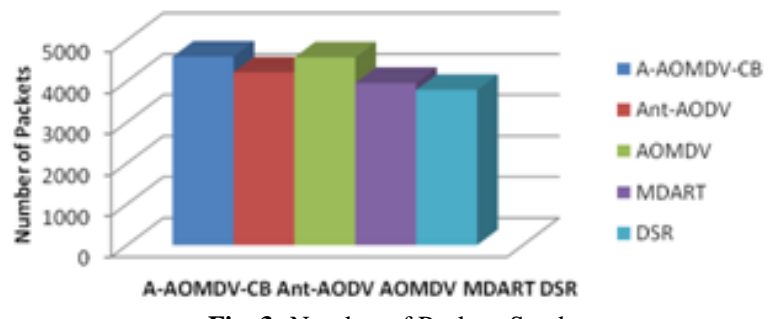

Fig. 3: Number of Packets Send

Figure 3 represents that A-AOMDV-CB has largest capacity to send the more data. All the other algorithms have lower value of this parameter than the value of the proposed algorithm.

\subsection{End to End Delay}

This performance parameter calculates the amount of time consumed by data packets in reaching to the target node. The lesser value of this parameter is preferred because lesser value predicts that the packet reach earlier to its destination.

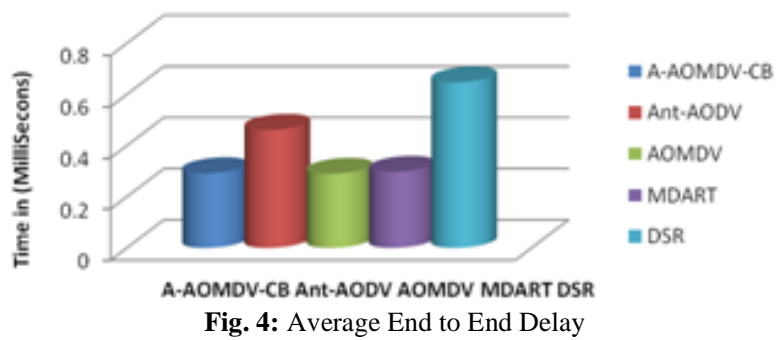

Figure 4 clearly indicates that AODV has the lowest value of this parameter as compared to other algorithms. A-AOMDV-CB has second lowest value when compared on the basis of average end to end delay.

\subsection{Jitter}

This parameter is calculated on the basis of difference between the end to end delays of the adjacent data packets. The parameter measures the drift in the normal value of delay. Jitter should be minimized in order to control the drift in normal value.

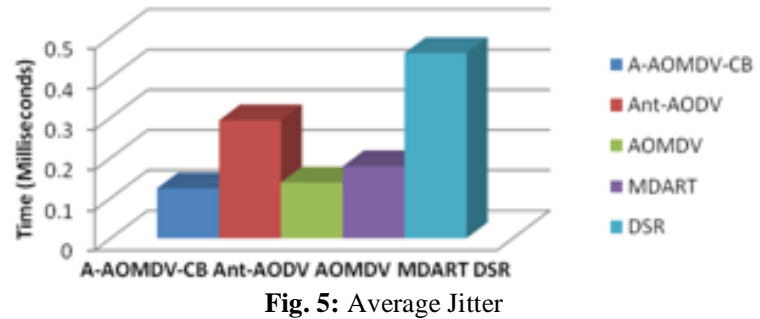

A-AOMDV-CB has the smallest jitter value as compared to other algorithms. So, new algorithm performs better when compared over the values of average jitter.

\subsection{Packet Drop}

Packets may be dropped due to packets collisions, congestion in network or on expiration of time to live value. The algorithm must drop the lesser number of packets in lieu to perform better.

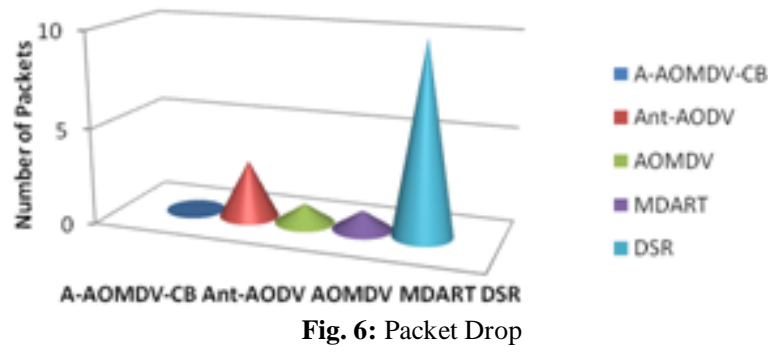

Graph shown above easily defines that lesser packets are dropped in A-AOMDV-CB as compared to other packets. So, new algorithm has better performance while measuring packet drop.

\subsection{Delivery Ratio}

Delivery ratio is calculated as the percentile in the loss of packet delivery. It can be measured by dividing the total packets lost to the overall packets send.

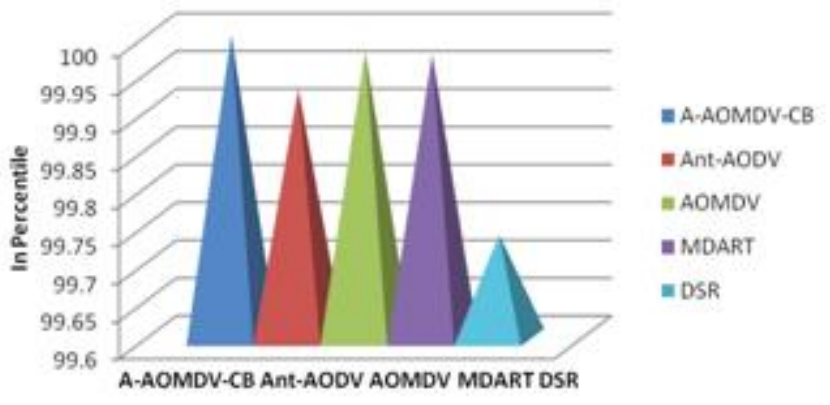

Fig. 7: Delivery Ratio

DSR has the lowest value of delivery ratio, whereas A-AOMDV$\mathrm{CB}$ has shown the highest value of delivery ratio. A-AOMDV-CB performs better than other algorithms of comparison.

\section{Conclusion}

This paper illustrates the work to improve the multipath routing in MANET using the technique of ACO. Many researchers have also worked in the area of multipath routing using ants' algorithms. The ants work as control packet in order to find the path and broadcast is being done by the intermediate nodes to their neighbors. This generates overhead in the network. To control this overhead, we have worked on the concept of controlled broadcast using concept of contention and back off technique. A-AOMDVCB is better than may DSR, Ant-AODV, AOMDV and MDART on comparing it on the ground of delivery ratio, average throughput, jitter, packet lost and number of packets send. However 
AOMDV performs lesser end to end delay than A-AOMDV-CB. But A-AOMDV-CB holds second place in evaluating packet delay. So, as an overall the new algorithm performs better than other protocols used for the comparison like Ant-AODV, DSR, AOMDV, MDART.

\section{References}

[1] S. Marwaha, C. K. Tham, D. Srinivasan, "Mobile Agents based Routing Protocol for Mobile Ad Hoc Networks", In proceeding of Department of Electrical and Computer Engineering, National University of Singapore

[2] R. R. Choudhary, S. Bhandhopadhyay and K. Paul, "A Distributed Mechanism for topology discovery in Ad Hoc Wireless Networks Using Mobile Agents," in Proceeding of Mobicom,pp. 145-146, 2000.

[3] D. S. Kumar, and R.S. Bhuvaneswaran," Proposal on Multi agent Ants based Routing Algorithm for Mobile Ad-Hoc Networks" IJCSNS International Journal of Computer Science and Network Security, Vol.7, No.6,2007.

[4] J. S. Baras, H. Mehta, ," A probabilistic emergent routing algorithm for mobile ad hoc networks"In WiOpt'03: Modeling and optimization in mobile, ad hoc and wireless networks, pp. 68-73,2003.

[5] G. D. Caro, M. Dorigo, "Ant colonies for adaptive routing in packet-switched communications networks" In Proceedings 5th international conference of parallel problem solving from nature. London: Springer.pp. 673-682, 1998 .

[6] M. Abolhasan, T. Wysocki, E. Dutkiewicz, "A review of routing protocols for mobile ad hoc networks". Adhoc Networks, Vol. 2(1) pp. 1-22, 2004.

[7] Y. Liu,"Advanced Dynamic Source Routing with QoS Guaran tee "'in the Proceedings of the Second Symposium International Computer Science and Computational Technology(ISCSCT '09) Huangshan, P. R. China, pp. 504-506, 2009.

[8] F. Ducatelle, G. D. Caro, L. M. Gambardella, "Ant agents for hybrid multipath routing in mobile ad hoc networks" In Proceedings of second annual conference on wireless on-demand network systems and services, pp. 44-53, 2009

[9] G. Singh, N. Kumar and A. K. Verma, "OANTALG: An Orientation Based Ant Colony Algorithm for Mobile Ad Hoc Networks", Wireless Personal Communication Springer, Vol. 77, Issue 3,pp. 1859-1884, 2014.

[10] S. Sharma, G. Singh, "Simulative Contemplation of AODV, AOMDV and MDART Protocols", International Journal of Advanced Engineering Research and Technology, Vol. 2, Issue 2,pp. 73-78,2014

[11] S. Marwaha, C.K. Tham, D. Srinivasan, "Mobile agents based routing protocol for mobile ad hoc networks," IEEE Global Communications Conference,pp.198-209, 2002.

[12] A. Kumar, R. Singh," Mobile ad hoc networks routing optimization techniques using swarm intelligence",In International Journal of Research in IT \& Management, Vol. 1(4), pp. 2231-4334, 2011.

[13] P. Sarala, D. Kalaiselvi, "Multipath Dynamic Source Routing with Cost and Ant Colony Optimization for MANETS", International Journal of Applied Engineering Research, Dindigul, Vol. 1, No 1 2010.

[14] M.R. Kumar, N. Geethanjali,"A Literature survey of Routing Protocols in MANETs, International journal of Science and Research, Vol. 2, 4,pp. 33-40,2013.

[15] A. Kaur, V. S. Dhaka, G. Singh , "ACO Agent based Routing in AOMDV Environment", International Conference on Advancements in Engineering \& Technology (ICAET-2016), MATEC Web of Conferences, Vol. 57,pp. 655-662,2016.

[16] S. Poornima, V.P. Muthu Kumar, "Comparision of AODV, OLSR, TORA Routing Protocols In MANET", International Journal of Modern Trends in Engineering and Research, Vol. 3, Issuec 2,2016.

[17] H. Zhang, X. Wang, P. Memarmoshrefi, D. Hogrefe, " A Survey of Ant Colony Optimization based Routing Protocols for Mobile Ad hoc Networks", IEEE Access, Volume PP, Issue 99, pp. 1-22, 12 , October, 2017.

[18] Y. B. Sim, S. G. Lee, S. Lee, " Function-Oriented Networking and On-Demand Routing System in Network Using Ant Colony Optimization Algorithm", Symmetry, Vollume 9 (11), pp 1-25, November 2017.

[19] A. Kaur, V. S. Dhaka, G. Singh, "Casting multipath behaviour into OANTALG to improve QoS”, IEEE Explore- International Conference on Computing for Sustainable Global Development, Pages 2076-2081, $16^{\text {th }}-18^{\text {th }}$ March, 2016.
[20] G. Ramprabu, S. Nagarajan, "Design and Analysis of Novel Modified Cross Layer Controller for WMSN", Indian Journal of Science and Technology, Vol 8(5), March 2015, pp.438-444. 